\title{
Newsfronts
}

\section{Helping Stem Cells Find Their Niche}

Researchers have reversed infertility in male mice using transplants of specialized cells involved in sperm formation, offering hope that a leading cause of male infertility may someday be reversible. Between 70 and 90\% of cases of male infertility arise from defects in spermatogenesis.

Sertoli cells, which compose the epithelial surface of the seminiferous tubules, nourish and support the germ cells, promoting their maturation into functional spermatozoa. Sertoli cells also provide what is known as a "niche" for the testicular stem cells that produce the germ cells, helping to maintain the stem cells in the undifferentiated state that is essential to their function.

Now, Ralph Brinster's group at the University of Pennsylvania (Philadelphia, PA) has announced the successful reestablishment of a niche, by means of the transplantation of functional murine Sertoli cells into infertile mice (Biol. Repr., November 2002). Steel/Steel ${ }^{d}$ mice produce defective Sertoli cells that are incapable of supporting spermatogenesis. When these mice were injected with dissociated testicular tissue from wild-type perinatal mice, between 30 and $40 \%$ of the recipients exhibited enhanced tubule development and restored spermatogenesis.

Before this procedure can become a viable option for male infertility treatment, it will be necessary to overcome a number of obstacles. Mature Sertoli cells no longer divide and cannot be expanded in culture, making it difficult to procure sufficient donor material. Furthermore, spermatogenesis in "restored" tubules is relatively inefficient, and further refinement will be necessary to maximize production of viable spermatozoa. Nonetheless, the amounts produced might be sufficient for intracytoplasmic sperm injection, a technique that, according to Brinster, is "very effective for achieving fertilization of human eggs when few spermatozoa are available."

Although the potential implications for infertility therapy are fairly obvious, Brinster sees greater possibilities in niche transplantation and its impact on the field of stem and germ cell research. He tells Lab Animal, "The ability to freeze stem cells allows long-term storage of any germline. In this context, transplantation and studies on the cells that support the process are enormously valuable."

- Michael Eisenstein

\section{Heart Drug Fights Paralysis in Mice}

Apart from fighting heart disease, the cholesterol-lowering drugs known as statins may also prevent and treat relapses of multiple sclerosis (MS). This debilitating disease, which causes loss of motor control and paralysis, affects as many as 2.5 million people worldwide.

MS is an autoimmune disease in which the body's immune cells, specifically helper T cells, attack myelin, the fatty tissue that covers and protects the nerves of the CNS. When myelin and the underlying nerves are destroyed, nerve impulses cannot be transmitted, causing the disabling symptoms of MS. At present, individuals have to take painful injections to relieve these symptoms. Now, a group led by Scott S. Zamvil of the University of California, San Francisco, report that the popular cholesterol-lowering drug atorvastatin (Lipitor), which can be taken in pill form, lessened or entirely sup- pressed the paralysis seen in a mouse model of MS (Nature, 7 November 2002).

Mice with experimental autoimmune encephalomyelitis (EAE) were fed atorvastatin once a day for several weeks. Zamvil's team saw that, in addition to abrogating symptoms, the drug reduced the amount of brain inflammation and CNS damage. They determined that atorvastatin inhibited the activation of $\mathrm{T}$ cells and the production of pro-inflammatory cytokines, leading to suppression of EAE. This is not the first use of statins in immunosuppression; they have also been useful in reducing rejection in heart transplant patients.

Although the results of the mouse study are promising, the authors still plan clinical trials to investigate the effects of atrovastatin in humans with MS. The drug may also prove effective in the treatment of other autoimmune diseases, such as diabetes and rheumatoid arthritis.

—Karen Zolnowski

\section{Watching Herpes Infection in Action}

Taking advantage of the molecule that gives fireflies their characteristic glow, a group of researchers at the Washington University School of Medicine (St. Louis, MO) has developed a noninvasive, real-time imaging

\section{Mouse Study Suggests New Risk Associated with Alzheimer's Vaccine}

Immunization with a vaccine against Alzheimer's disease (AD) doubled the risk of stroke in mice. This finding may help explain the brain inflammation seen recently in human subjects participating in tests of a highly touted vaccine against this dreaded neurodegenerative disease.

A group led by Mathias Jucker at the University of Basel (Switzerland) injected a transgenic mouse model of AD weekly with a monoclonal antibody that recognizes $\beta$-amyloid, the peptide that accumulates in the brains of $\mathrm{AD}$ patients (Science, 15 November 2002). After five months, the immunized mice exhibited a $23 \%$ decrease in amyloid load but were also more than twice as likely to suffer cerebral hemorrhage as were mice in the control group.

These microhemorrhages occurred only in areas of the brain affected by cerebral amyloid angiopathy (CAA), which is characterized by the intravascular deposition of amyloid protein. Researchers believe this accumulation of protein weakens the vessel walls, making them more susceptible to hemorrhage. Although CAA appears in $80 \%$ of AD patients, the APP23 mouse strain used in this study is the only model of AD that exhibits this condition, possibly explaining why this is the first mouse study to uncover this potentially fatal side effect of the vaccine.

-Tanja Schub 